\title{
Clinical characteristics and risk factors of catheter-associated urinary tract infections caused by Klebsiella Pneumoniae
}

\author{
Xiaolei Liu ${ }^{1 \#}$, Fangfang Sai ${ }^{2 \#}$, Lanyu Li ${ }^{1}$, Changqing Zhu ${ }^{1}$, Huan Huang ${ }^{1}$ \\ ${ }^{1}$ Department of Emergency Medicine, Renji Hospital, School of Medicine, Shanghai Jiaotong University, Shanghai, China; ${ }^{2}$ Department of \\ Geriatrics, Renji Hospital, School of Medicine, Shanghai Jiaotong University, Shanghai, China \\ Contributions: (I) Conception and design: H Huang; (II) Administrative support: C Zhu; (III) Provision of study materials or patients: H Huang; (IV) \\ Collection and assembly of data: X Liu, F Sai; (V) Data analysis and interpretation: X Liu, L Li; (VI) Manuscript writing: All authors; (VII) Final \\ approval of manuscript: All authors. \\ "These authors contributed equally to this work. \\ Correspondence to: Huan Huang, MD. Department of Emergency Medicine, Renji Hospital, School of Medicine, Shanghai Jiaotong University, 160\# \\ Pujian Rd, Pudong New District, Shanghai 200127, China. Email: Dr_huan@163.com.
}

Background: The clinical characteristics and risk factors of catheter-associated urinary tract infections (CAUTIs) caused by Klebsiella pneumoniae (KP) have not been well investigated.

Methods: This retrospective study performed at a university teaching hospital in China from January 2012 to November 2017 analyzed data for 227 patients with urinary tract infection (UTI) caused by KP. Patients' demographic characteristics and clinical outcomes were recorded. Risk factors were analyzed using a binary logistic regression model.

Results: Of 227 patients with Klebsiella pneumoniae-related urinary tract Infection (KP-UTI), the infection was catheter-associated in 90 patients. More than half of them were male (60\%), over 60 years old, hospitalized in general ward, always acquired in hospital, and got a longer hospitalization more than one month. The Klebsiella pneumoniae-related catheter-associated urinary tract infections (KP-CAUTIs) patients always combined with lots of chronic comorbidities. A high proportion of invasive device, extendedspectrum $\beta$-lactamase (ESBL) expression and multidrug resistance (MDR) were found in KP-CAUTIs patients. When taken antimicrobial activity into consideration, $K P$-CAUTIs patients performed resistance to most antibiotics in varying degrees. Logistic regression analysis revealed that after grouping by ESBL expression and in-hospital mortality among patients with KP-CAUTI, complicated urinary tract infection (cUTI) was an independent risk factor for ESBL positive KP-CAUTIs [odds ratio (OR) 59.256; 95\% CI, 3.417-1,027.628; $\mathrm{P}=0.005]$, whereas congestive heart failure was identified as an independent risk factor for in-hospital mortality (OR 25.592; 95\% CI, 2.376-275.629; P=0.008) in KP-CAUTI patients.

Conclusions: Patients with KP-CAUTI displayed distinctive characteristics. cUTI and congestive heart failure were independently associated with ESBL expression and in-hospital mortality in patients with $K P$ CAUTI.

Keywords: Catheter-associated urinary tract infections (CAUTIs); Klebsiella pneumoniae (KP); extended-spectrum $\beta$-lactamase (ESBL); mortality; risk factors

Submitted Apr 30, 2020. Accepted for publication Aug 10, 2020.

doi: 10.21037/apm-20-1052

View this article at: http://dx.doi.org/10.21037/apm-20-1052 


\section{Introduction}

Urinary tract infections (UTIs) represent $12.9 \%$ of healthcare-associated infections and $23 \%$ of infections in the intensive care unit (ICU), with approximately $70 \%$ of them being catheter-associated (CAUTIs) (1-3). CAUTIs occur at a rate of $3-10 \%$ per day of catheterization and approach a frequency of $100 \%$ within 30 days of hospitalization (4). The onset of CAUTIs can significantly affect patients' clinical outcomes, including a longer stay in the hospital, higher healthcare expenditures, and the overuse of antibiotics, as well as the possibility of higher mortality rates (5-7).

The presence of a catheter creates a special environment for bacteria colonization and biofilm formation, which increase the risk of infection and weaken the efficacy of treatment (8). Klebsiella pneumoniae (KP), as a normal flora of the gastrointestinal tract, often causes UTIs through cross-transmission (9). In many studies, $K P$ was identified as the second most prevalent pathogen following by Escherichia coli among Enterobacteriaceae in UTIs (8-10). As a result of the indiscriminate and widespread use of antibiotics, $K P$ produces high levels of extended-spectrum $\beta$-lactamase (ESBL), which causes resistance to most antibiotics excluding carbapenems. However, multidrugresistant isolates of $K P$, including resistant to carbapenems isolates, have been detected recently, leading to the failure of empirical therapy and inevitably increased mortality (11).

Although Ikeda and colleagues reported a nosocomial infection outbreak of ESBL producing $K P$ in patients with CAUTI in 2018, little is known regarding Klebsiella pneumoniae-related catheter-associated urinary tract infections (KP-CAUTIs) (12). Our study aimed to summarize the clinical characteristics and identify the risk factors of KP-CAUTIs to improve our understanding of this infection.

We present the following article in accordance with the STROBE Checklist (available at http://dx.doi. org/10.21037/apm-20-1052).

\section{Methods}

\section{Study design}

A retrospective study of Chinese patients with Klebsiella pneumoniae-related urinary tract Infection (KP-UTI) was conducted in Renji Hospital affiliated with the Shanghai Jiaotong University of Medicine, an 1800-bed tertiary care university teaching hospital in Shanghai, China. The study period was January 1, 2012 to November 31, 2017. Clinical manifestations were determined from medical charts. The study was observational in that the administration of antimicrobial agents and therapeutic managements were controlled by patients' physicians, and not by the investigators.

We collected records for all patients with a urine culture yielding $K P$ levels $>10^{5}$ colony-forming units $/ \mathrm{ml}$ with only one microorganism present, as well as a diagnosis of UTI by clinicians. The exclusion criteria were as follows: (I) outpatients; (II) patients less than 18 years old at the time of admission; (III) recurrent episodes (only the first instance of a positive $K P$ urine culture in patients with UTI was included in our study); (IV) a history of hospitalization for more than $24 \mathrm{~h}$ within the 90 days before the index hospitalization; (V) hospital stay exceeding 6 months; and (VI) inpatients with incomplete or unavailable medical records.

\section{Definitions}

The onset of UTI was defined as the date on which the first $K P$-positive urine sample was collected. CAUTI was diagnosed using only urine cultures collected more than 2 days after catheterization. Non-CAUTI was defined by positive urine cultures in patients who did not undergo catheterization. Hospital-acquired UTI were defined as infection in which the first positive culture was obtained more than $48 \mathrm{~h}$ after hospital admission or within $48 \mathrm{~h}$ after discharge from the hospital. Complicated UTI (cUTI) was originally defined by the US Food and Drug Administration (FDA) as the presence of at least one of the following findings: indwelling urinary catheter, urinary retention, neurogenic bladder, obstructive uropathy, renal impairment caused by intrinsic renal disease, renal transplantation, urinary tract modifications, or pyelonephritis with normal urinary tract anatomy (13). To further understand CAUTIs in our research, we defined cUTI as UTI or CAUTI combined with at least one of the following findings: urinary retention, neurogenic bladder, obstructive uropathy, renal impairment caused by intrinsic renal disease, renal transplantation, urinary tract modification, or pyelonephritis with normal urinary tract anatomy. The duration of catheterization was defined as length between the date of catheterization and that on which the first positive urine culture was collected. Length of stay (LOS) was defined as time from admission until discharge or death in the hospital. In-hospital mortality was defined as death of 
any cause during hospitalization.

Clinical variables examined in patients with $K P$-UTI included age, gender, hospitalized ward, comorbidities, insertion of invasive devices, and laboratory findings., multidrug resistance (MDR) was defined as acquired resistance to three or more of the following antimicrobial classes: aminoglycosides (gentamicin, tobramycin or amikacin); carbapenems (imipenem); first-/secondgeneration (cefazolin) and third-/fourth-generation (cefotaxime or ceftazidime) cephalosporins; fluoroquinolones (ciprofloxacin or levofloxacin or norfloxacin); tetracycline; ampicillin-sulbactam; chloramphenicol; and trimethoprim-sulphamethoxazole. Carbapenemresistant Enterobacteriaceae (CRE) was defined as an Enterobacteriaceae isolate that was resistant to carbapenems. Bloodstream infections (BSIs) by KP (KP-BSIs) were defined as the presence of $K P$ in blood cultures in patients with simultaneous $K P$-UTI.

\section{Microbiology}

$K P$ isolates were identified using the Vitek 2 Advanced Expert System (bioMèrieux, Marcy l'Etoile, France), and antibiotic susceptibility was tested using the Kirby-Bauer agar disk diffusion method. Antibiotic susceptibility was interpreted according to the European Committee on Antimicrobial Susceptibility Testing guidelines (14).

The study was conducted in accordance with the Declaration of Helsinki (as revised in 2013). The study was approved by the Renji Hospital Ethics Committee (Shanghai Jiaotong University School of Medicine) (NO. [2018]231) and individual consent for this retrospective analysis was waived.

\section{Statistical analysis}

Student's $t$-test was used to compare continuous variables, and the chi-squared test or Fisher's exact test was used to compare categorical variables. A stepwise logistic regression model was applied to identify independent risk factors for KP-CAUTIs in subgroup analysis. Risk factors with $\mathrm{P}$ values less than 0.10 in the univariate analysis were included in the initial model, and forward stepwise selection was performed to develop the final model. A $\mathrm{P}$ value less than 0.05 was considered statistically significant. All data were analyzed using IBM SPSS Statistics for Windows (version 22.0). Odds ratios (ORs) and $95 \%$ CIs were calculated to evaluate the strength of any association.

\section{Results}

\section{Clinical characteristics of KP-CAUTIs}

A total of 227 patients with $K P$-UTI were identified during the study period, including 90 patients with catheter. The $K P$-CAUTIs patients represented their unique clinical characteristics. More than half of them were male $(60 \%)$, over 60 years old, hospitalized in general ward, always acquired in hospital, and got a longer hospitalization more than one month. The KP-CAUTIs patients frequently combined with lots of chronic comorbidities, such as hypoproteinemia, solid tumor, and congestive heart failure. More than half of $K P$-CAUTIs patients were equipped with invasive devices such as central venous catheter and thoracic and abdominal drainage tube. ESBL expression and MDR were also found in them. When taken antimicrobial activity into account, $K P$-CAUTIs patients performed resistance to most antibiotics in varying degrees (Table 1).

\section{Comparison of patients with KP-CAUTI subgrouped by ESBL expression}

We divided all 90 patients with KP-CAUTI into two subgroups according to the presence or absence of ESBL. Compared with the ESBL positive subgroup $(n=58)$, significant differences were observed regarding the rates of ICU occupancy, hospital acquisition, combined with cUTI, duration of catheterization, and LOS in the hospital in the ESBL negative group $(\mathrm{P}<0.001$, $\mathrm{P}<0.001, \mathrm{P}=0.021, \mathrm{P}<0.001$, and $\mathrm{P}=0.001$, respectively). Regarding comorbidities, the ESBL positive subgroup was more likely to present with congestive heart failure, cerebrovascular accident, and septic shock, as well as lower rates of leukemia/lymphoma $(\mathrm{P}=0.003, \mathrm{P}=0.026, \mathrm{P}=0.013$, and $\mathrm{P}=0.042$, respectively). The ESBL positive subgroup also exhibited obvious differences in the rates of invasive device use and occurrence of MDR and CRE (all $\mathrm{P}<0.005$, Table 2).

Multiple potential risk factors were included in multivariate logistic regression analysis. ESBL positive $K P$ CAUTIs were found to be significantly associated with cUTI (OR 59.256; 95\% CI, 3.417-1027.628; P=0.005), and a trend toward a correlation between central venous catheterization and $K P$-CAUTIs was discovered (OR 63.648; 95\% CI, 0.973-4,163.155; $\mathrm{P}=0.052$ ) (Table 3).

\section{Comparison of patients with KP-CAUTI subgrouped by in-hospital mortality or not}

We divided all patients with KP-CAUTI into two 
Table 1 Clinical characteristics of KP-CAUTIs patients

\begin{tabular}{|c|c|}
\hline Characteristic & $\mathrm{N}=90$ \\
\hline \multicolumn{2}{|l|}{ Age (year), n (\%) } \\
\hline $18-45$ & $6(6.7)$ \\
\hline $46-60$ & $19(21.1)$ \\
\hline $61-75$ & $32(35.6)$ \\
\hline$>75$ & $33(36.7)$ \\
\hline Gender (male), n (\%) & $54(60.0)$ \\
\hline \multicolumn{2}{|l|}{ Hospitalization ward, n (\%) } \\
\hline General ward & $63(70.0)$ \\
\hline ICU & $27(30.0)$ \\
\hline Hospital acquired UTIs, n (\%) & $65(72.2)$ \\
\hline Complicated UTIs, n (\%) & $31(34.4)$ \\
\hline Length of stay (mean \pm SD) (day) & $38.34 \pm 42.83$ \\
\hline In-hospital mortality, n (\%) & $14(15.6)$ \\
\hline \multicolumn{2}{|l|}{ Comorbidities, n (\%) } \\
\hline Diabetes mellitus & $17(18.9)$ \\
\hline Chronic respiratory disease & $18(20.0)$ \\
\hline Chronic liver disease & $8(8.9)$ \\
\hline Chronic kidney disease & $24(26.7)$ \\
\hline Congestive heart failure & $32(35.6)$ \\
\hline Solid tumor & $36(40.0)$ \\
\hline Leukemia/lymphoma & $3(3.3)$ \\
\hline Cerebrovascular accident & $17(18.9)$ \\
\hline Septic shock & $22(24.4)$ \\
\hline Immunosuppression & $14(15.6)$ \\
\hline Hypoproteinemia & $52(57.8)$ \\
\hline \multicolumn{2}{|l|}{ Invasive device, n (\%) } \\
\hline Central venous catheter & $49(54.4)$ \\
\hline Ventilation & $38(42.2)$ \\
\hline Nasogastric feeding tube & $43(47.8)$ \\
\hline Thoracic and abdominal drainage tube & $48(53.3)$ \\
\hline
\end{tabular}

Table 1 (continued)

\begin{tabular}{|c|c|}
\hline Characteristic & $\mathrm{N}=90$ \\
\hline \multicolumn{2}{|l|}{ Lab finding } \\
\hline WBC (mean $\pm \mathrm{SD}),\left(\times 10^{9} / \mathrm{L}\right)$ & $9.78 \pm 4.67$ \\
\hline $\mathrm{BPC}($ mean $\pm \mathrm{SD}),\left(\times 10^{9} / \mathrm{L}\right)$ & $210.17 \pm 103.13$ \\
\hline Hemoglobin (mean $\pm \mathrm{SD}$ ), (g/L) & $107.84 \pm 27.75$ \\
\hline Albumin (mean $\pm \mathrm{SD}),(\mathrm{g} / \mathrm{L})$ & $33.21 \pm 7.85$ \\
\hline $\mathrm{CRP}($ mean $\pm \mathrm{SD}),(\mathrm{mg} / \mathrm{L})$ & $45.31 \pm 44.29$ \\
\hline PCT (mean \pm SD), (ng/mL) & $2.39 \pm 7.85$ \\
\hline ESR (mean $\pm \mathrm{SD}),(\mathrm{mm} / \mathrm{h})$ & $37.39 \pm 31.1$ \\
\hline Creatine (mean $\pm \mathrm{SD}),(\mathrm{mmol} / \mathrm{L})$ & $118.06 \pm 155.82$ \\
\hline Blood glucose (mean $\pm \mathrm{SD}),(\mathrm{mmol} / \mathrm{L})$ & $7.22 \pm 6.23$ \\
\hline ESBL (+), n (\%) & $58(64.4)$ \\
\hline MDR (+), n (\%) & $61(67.8)$ \\
\hline CRE (+), n (\%) & $27(30.0)$ \\
\hline Bloodstream infection with KP, n (\%) & $3(3.3)$ \\
\hline \multicolumn{2}{|c|}{ Antimicrobial activity of KP (resistance), $\mathrm{n}(\%)$} \\
\hline Second generation cephalosporins & $64(71.1)$ \\
\hline Third generation cephalosporins & $51(56.7)$ \\
\hline Forth generation cephalosporins & $51(56.7)$ \\
\hline Cefoperazone-sulbactam & $53(58.9)$ \\
\hline Aminoglycosides & $41(45.6)$ \\
\hline Fluoroquinolones & $56(62.2)$ \\
\hline Carbapenems & $27(30.0)$ \\
\hline Piperacillin-Tazobactam & $74(82.2)$ \\
\hline
\end{tabular}

KP-CAUTIs, Klebsiella pneumoniae-related catheter-associated urinary tract infection; WBC, white blood cell, BPC, blood platelet cell, CRP, C-reactive protein, PCT, procalcitonin, ESR, erythrocyte sedimentation rate; ESBL, extended-spectrum $\beta$-lactamase; MDR, multidrug resistance; CRE, Carbapenemresistant Enterobacteriaceae.

Table 1 (continued) 
Table 2 Risk factors analysis of ESBL positive KP-CAUTIs

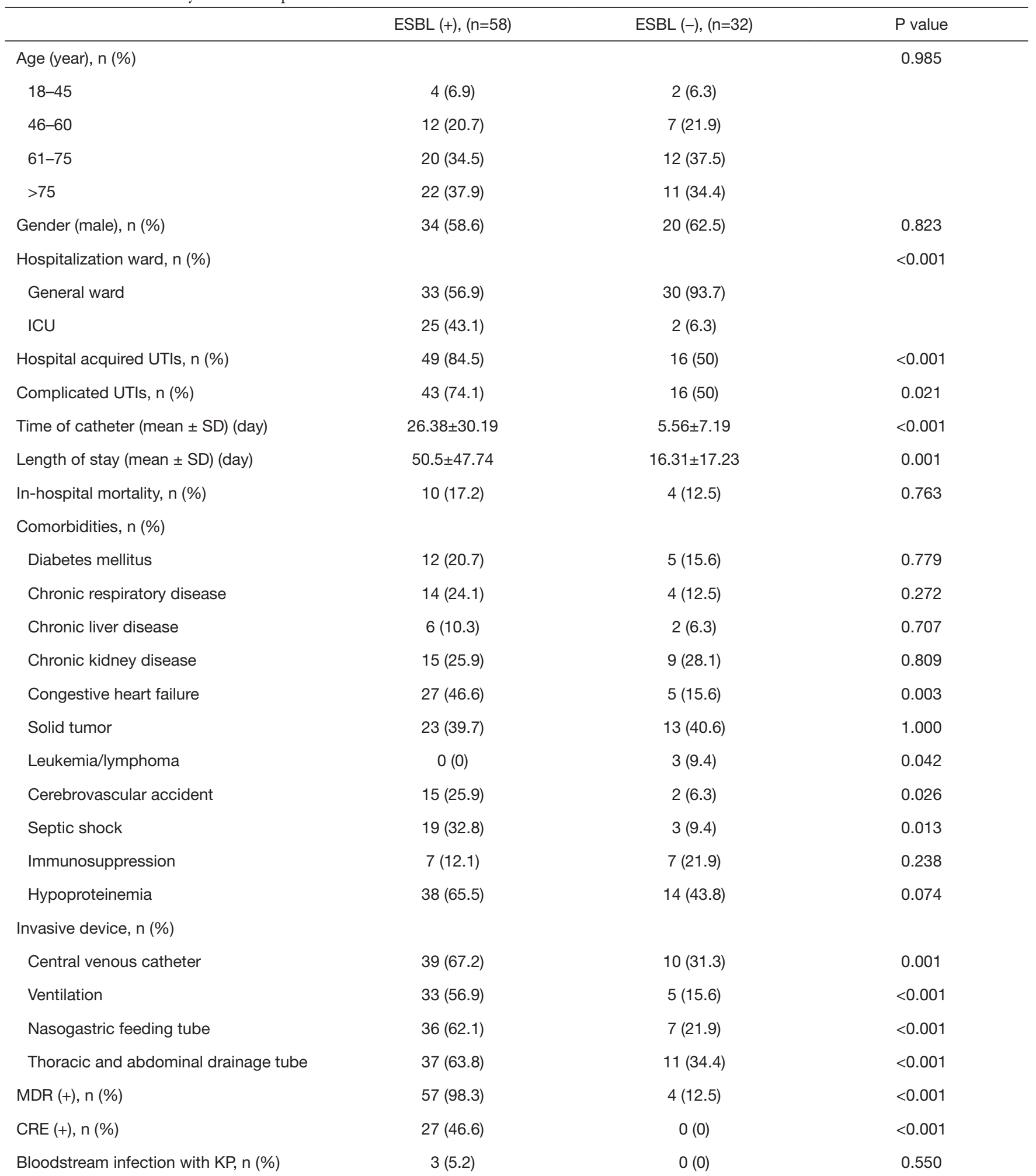

ESBL, extended-spectrum $\beta$-lactamase; KP-CAUTIs, Klebsiella pneumoniae-related catheter-associated urinary tract infection; MDR, multidrug resistance; $\mathrm{CRE}$, Carbapenem-resistant Enterobacteriaceae. 
Table 3 Multivariate logistic regression analysis of ESBL positive KP-CAUTIs

\begin{tabular}{lcc}
\hline & OR $(95 \% \mathrm{Cl})$ & $\mathrm{P}$ \\
\hline Complicated UTIs, $\mathrm{n}(\%)$ & $59.256(3.417-1,027.628)$ & 0.005 \\
Central venous catheter & $63.648(0.973-4,163.155)$ & 0.052 \\
\hline
\end{tabular}

ESBL, extended-spectrum $\beta$-lactamase; KP-CAUTIs, Klebsiella pneumoniae-related catheter-associated urinary tract infections.

subgroups according to death in hospital or not, and relevant conditions were compared via univariate analysis. Fourteen patients died during hospitalization. Between the two subgroups, significant differences were found regarding the ICU occupancy rate, frequency of combined cUTI, duration of catheterization, and LOS $(\mathrm{P}=0.004$, $\mathrm{P}=0.019, \mathrm{P}=0.008$, and $\mathrm{P}=0.001$, respectively). Regarding comorbidities, the in-hospital mortality subgroup had higher rates of congestive heart failure and septic shock $(\mathrm{P}<0.001$ and $\mathrm{P}=0.001$, respectively). Patients in the inhospital mortality subgroup were more likely to use invasive devices and exhibit infection with CRE (both $\mathrm{P}=0.002$ ). Concerning antimicrobial activity, the subgroups only displayed a difference in the frequency of resistance to carbapenems $(\mathrm{P}=0.002$, Table 4).

According to multivariate logistic regression analysis, only congestive heart failure was significantly associated with in-hospital mortality in patients with KP-CAUTI (OR 25.592; 95\% CI, 2.376-275.629; $\mathrm{P}=0.008$ ) (Table 5).

\section{Discussion}

Although $K P$ has had an increasingly important role in infection at various sites (i.e., urinary tract, pulmonary, bloodstream, biliary tract) in recent years, there has been little research on CAUTIs caused by $K P(9,11,15,16)$. To our knowledge, this is the first population-based study of $K P$-CAUTIs in China.

Urinary catheterization has long been recognized as a major risk factor for healthcare-associated UTIs. CAUTIs are amongst the most common nosocomial infections, and they are also considered among the most common complications associated with indwelling urinary catheters (17). Female was identified as an independent risk factor for CAUTIs in a study by Gillen et al. because of their shorter and wider urethra (18). However, Hagerty et al. did not find the same relevance in their study (19). In our study, more than half of patients with $K P$-CAUTI were male, which differs from previous findings. This may be explained by their underlying or functional urethra obstruction with age makes catheterization necessary, which may change the microbial environment of urine, resulting in biofilm formation, and emergence of drug-resistant bacteria. Catheterization always results in a higher rate of ICU admission and longer LOS, which are linked to substantial morbidity and mortality. In the US, approximately $60 \%$ of ICU and $20 \%$ of general ward patients undergo catheterization, which increases the risk of hospital-acquired CAUTIs $(20,21)$. In addition, CAUTIs were linked to 2.4 additional inpatient days among pediatric patients in Goudie's study. Similar findings were reported in adult patients by Clarence, who found that CAUTIs increased LOS in the ICU and hospital by a median of 13 and 2.4 days, respectively $(5,22)$. Clarence also identified an association between CAUTIs and increased crude hospital mortality even after restricting the analysis (5). A nearly 3 -fold increase in mortality among hospitalized patients with CAUTI was reported by Platt (23). The most commonly cited indications for catheter use were the need for accurate input or output monitoring in critically ill patients, perioperative use, and prolonged immobilization (24). Catheterization is extremely necessary among patients with fatal or bedridden comorbidities such as congestive heart failure, septic shock, or cerebrovascular accident, which always present with consumptive malnutrition as reported in our study. Both $\mathrm{Li}$ and Loveday identified diabetes mellitus and chronic kidney diseases as high risk factors for CAUTIs for possible kidney injury and infection $(25,26)$; however, our data did not reveal such tendency. This may be related to differences in study designs and populations, but their potential mechanisms are worthy of further study. What we can confirm is that patients who exhibit prolonged LOS in the hospital after catheterization usually have serious underlying comorbidities which make them more susceptible to infections as well as increased antibiotic resistance and mortality.

ESBL is an enzyme usually produced by Enterobacteriaceae species that makes them resistant to several antibiotics commonly used to treat UTIs (27). In a program of 199 hospitals from 42 countries worldwide conducted by Castanheira et al. over 20 years, the second most common isolate with an ESBL phenotype was $K P(43.7 \%)$. They also found that the most common multidrug-resistant species was $K P(35.2 \%)$, and carbapenem-resistant $K P$ was found to be the main driver of CRE positivity, comprising $71.1 \%$ of such isolates. These isolates were linked to delayed diagnosis and management, increased ICU admission, high 
Table 4 Risk factors analysis of in-hospital mortality in patients with KP-CAUTI

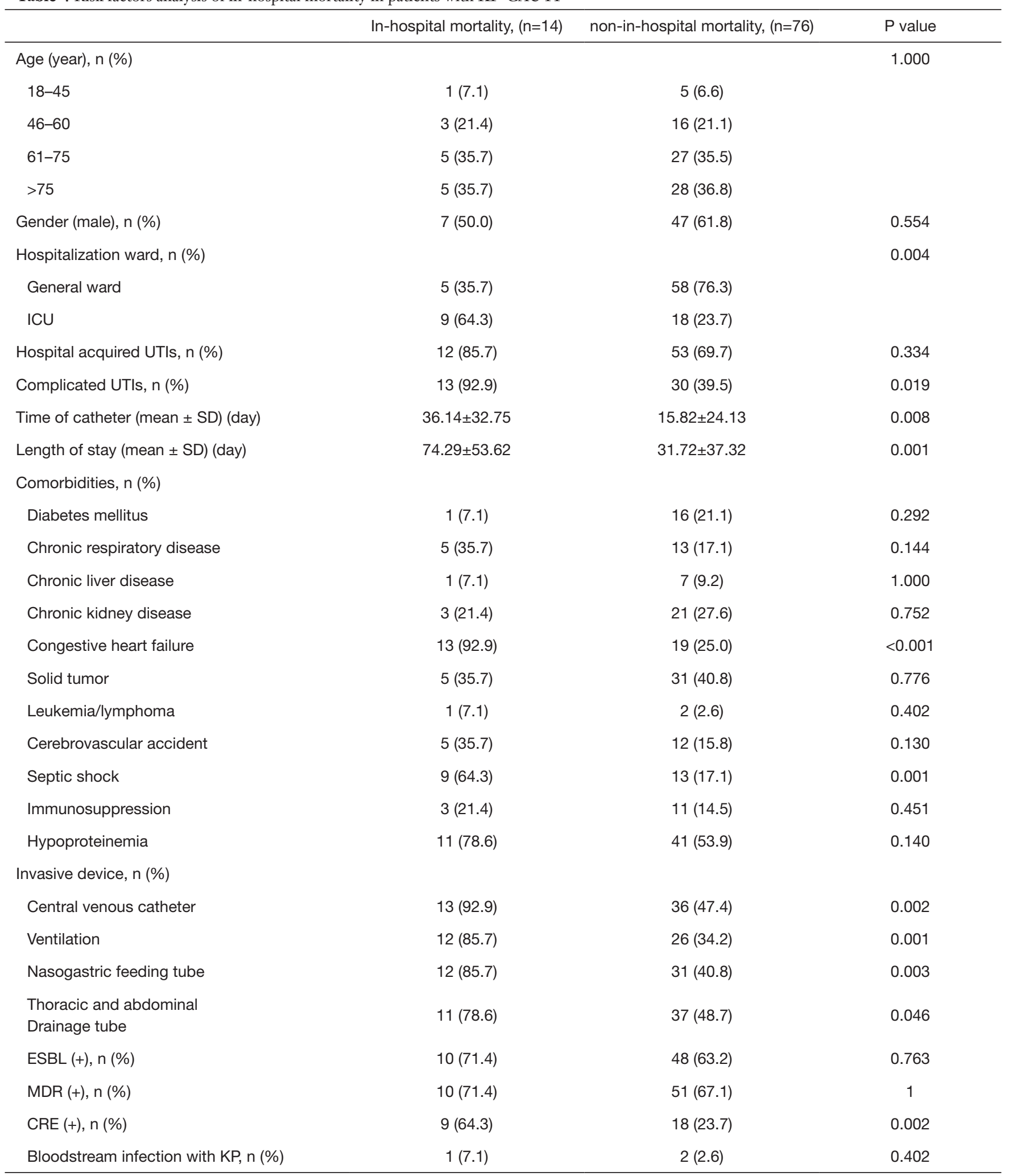

Table 4 (continued) 
Table 4 (continued)

\begin{tabular}{|c|c|c|c|}
\hline & In-hospital mortality, $(n=14)$ & non-in-hospital mortality, $(n=76)$ & $P$ value \\
\hline Third generation Cephalosporins & $10(71.4)$ & $41(53.9)$ & 0.257 \\
\hline Forth generation Cephalosporins & $11(78.6)$ & $40(52.6)$ & 0.085 \\
\hline Aminoglycosides & $9(64.3)$ & $32(42.1)$ & 0.152 \\
\hline Fluoroquinolones & $9(64.3)$ & $47(61.8)$ & 1 \\
\hline Carbapenems & $9(64.3)$ & $18(23.7)$ & 0.002 \\
\hline Piperacillin-Tazobactam & $13(92.9)$ & $61(80.3)$ & 0.450 \\
\hline
\end{tabular}

KP-CAUTI, Klebsiella pneumoniae-related catheter-associated urinary tract infection; ESBL, extended-spectrum $\beta$-lactamase; MDR, multidrug resistance; CRE, Carbapenem-resistant Enterobacteriaceae.

Table 5 Multivariate logistic regression analysis of in-hospital mortality in patients with KP-CAUTI

\begin{tabular}{lcc}
\hline & OR $(95 \% \mathrm{Cl})$ & $\mathrm{P}$ \\
\hline Congestive heart failure & $25.592(2.376-275.629)$ & 0.008 \\
\hline $\begin{array}{l}\text { KP-CAUTI, Klebsiella pneumoniae-related catheter-associated } \\
\text { urinary tract infection. }\end{array}$
\end{tabular}

mortality, increased hospital cost, and longer LOS in the hospital $(27,28)$. Both Ranjan Dash and Søraas reported that diabetes mellitus is significantly associated with ESBL expression in patients with UTI. However, we did not obtain similar results in our study, which may be the result of our focus on KP-CAUTIs opposed to a broader analysis of patients with UTI $(29,30)$. An in-depth and specific study should be conducted to clarify the mechanisms. cUTI, as defined by the FDA, applies to pyelonephritis or UTI in a host with predisposing conditions that have been associated with high rates of treatment failure and serious complications, especially relapse and the development of antibiotic resistance $(14,31,32)$. As a type of cUTIs by FDA, CAUTIs always arise in patients with critical illness, perioperative state, or prolonged immobilization. To deeply study CAUTIs, we focused on patients with CAUTI and other structural urinary tract disorders separately for further comparison. In our study, cUTI was distinctively related to ESBL positive KP-CAUTIs. This may be attributable to abnormal urination, prolonged urinary retention, and microbial culturing, which increase the risk of antibiotic resistance. Additional studies are needed to gain insights into their underlying relationships. Interestingly, we found an underlying correlation between central venous catheterization and ESBL positive KP-CAUTIs in our subgroup analysis. Central venous catheterization, which had not previously been studied, was cited as a risk factor for nosocomial bacteremia secondary to UTIs in a study by Sante providing a foundation for further investigation (33).

In-hospital mortality is the utmost worst prognosis for patients, every link of diagnosis and treatment process can influence disease trend even lead to medical treatment failure eventually. Several variables, including age, disease state, indwelling with catheter, LOS, microbial infection, or antibiotic resistance, can increase the risk of death. However, only congestive heart failure was identified as an independent risk factor for death in hospital among patients with $K P$ CAUTI in our study. As a fatal disease itself, congestive heart failure requires catheterization for accurately monitoring input and output. The catheter itself and duration of catheterization both increase the risk of nosocomial death. However, in a study by Allison, congestive heart failure was surprisingly associated with a lower risk of CAUTIs in their population (34). The reason for this conclusion may be possibly explained by differences in the populations (age, sample size, country, and setting). Further studies with larger multicenter samples are needed to confirm this correlation.

There were several limitations in our study. Firstly, our data were obtained from a single hospital, which resulted in a less diverse and representative population of patients. Secondly, our study was retrospective, carrying a certain risk of bias. Thirdly, the data were obtained from a 
hospital in southeastern China, and the findings may not be generalizable to other regions.

\section{Conclusions}

Our study provided new insights into CAUTIs, as clinical characteristics and risk factors for this infection were identified. Patients with KP-CAUTI exhibited unique features. cUTI was identified as an independent risk factor for ESBL positive $K P$-CAUTIs, and congestive heart failure was identified as an independent risk factor for in-hospital mortality among patients with KP-CAUTI. We believe this article will provide meaningful information for clinicians in daily practice, and additional large-scale, multicenter studies are needed to investigate this special group.

\section{Acknowledgments}

Funding: None.

\section{Footnote}

Reporting Checklist: The authors have completed the STROBE Checklist. Available at http://dx.doi.org/10.21037/ apm-20-1052

Data Sharing Statement: Available at http://dx.doi. org/10.21037/apm-20-1052

Peer Review File: Available at http://dx.doi.org/10.21037/ apm-20-1052

Conflicts of Interest: All authors have completed the ICMJE uniform disclosure form (available at http://dx.doi. org/10.21037/apm-20-1052). The authors have no conflicts of interest to declare.

Ethical Statement: The authors are accountable for all aspects of the work in ensuring that questions related to the accuracy or integrity of any part of the work are appropriately investigated and resolved. The study was conducted in accordance with the Declaration of Helsinki (as revised in 2013). The study was approved by the Renji Hospital Ethics Committee (Shanghai Jiaotong University School of Medicine) (NO. [2018]231) and individual consent for this retrospective analysis was waived.

Open Access Statement: This is an Open Access article distributed in accordance with the Creative Commons Attribution-NonCommercial-NoDerivs 4.0 International License (CC BY-NC-ND 4.0), which permits the noncommercial replication and distribution of the article with the strict proviso that no changes or edits are made and the original work is properly cited (including links to both the formal publication through the relevant DOI and the license). See: https://creativecommons.org/licenses/by-nc-nd/4.0/.

\section{References}

1. Magill SS, Edwards JR, Bamberg W, et al. Multistate point-prevalence survey of health care-associated infections. N Engl J Med 2014;370:1198-208.

2. Chenoweth CE, Saint S. Urinary tract infections. Infect Dis Clin North Am 2016;30:869-85.

3. Burton DC, Edwards J, Srinivasan A, et al. Trends in catheter-associated urinary tract infection in adult intensive care units-United States, 1990-2007. Infect Control Hosp Epidemiol 2011;32:748-56.

4. Assadi F. Strategies for Preventing Catheter-associated Urinary Tract Infections. Int J Prev Med 2018;9:50.

5. Chant C, Smith OM, Marshall JC, et al. Relationship of catheter-associated urinary tract infection to mortality and length of stay in critically ill patients: a systematic review and meta-analysis of observational studies. Crit Care Med 2011;39:1167-73.

6. Hooton TM, Bradley SF, Cardenas DD, et al. Diagnosis, prevention, and treatment of catheter-associated urinary tract infection in adults: 2009 international clinical practice guidelines from the Infectious Diseases Society of America. Clin Infect Dis 2010;50:625-63.

7. Kazi MM, Harshe A, Sale H, et al. Catheter associated urinary tract infections (CAUTI) and antibiotic sensitivity pattern from confifirmed cases of CAUTI in a tertiary care hospital: a prospective study. Clin Microbiol 2015;4:193.

8. Al Yousef SA, Younis S, Farrag E, et al. Clinical and Laboratory Profile of Urinary Tract Infections Associated with Extended Spectrum $\beta$-Lactamase Producing Escherichia coli and Klebsiella pneumoniae. Ann Clin Lab Sci 2016;46:393-400.

9. Kader AA, Angamuthu K. Extended-spectrum beta-lactamases in urinary isolates of Escherichia coli, Klebsiellapneumoniaeand other gram-negative bacteria in a hospital in Eastern Province, Saudi Arabia. Saudi Med J 2005;26:956-9.

10. Albu S, Voidazan S, Bilca D, et al. Bacteriuria and asymptomatic infection in chronic patients with indwelling urinary catheter: The incidence of ESBL bacteria. 
Medicine (Baltimore) 2018;97:e11796.

11. Mazzariol A, Bazaj A, Cornaglia G. Multi-drug-resistant Gram-negative bacteria causing urinary tract infections: a review. J Chemother 2017;29:2-9.

12. Ikeda Y, Shigemura K, Nomi M, et al. Infection Control Following an Outbreak of Extended-Spectrum BetaLactamase-Producing Klebsiella pneumoniae Isolated from Catheter-Associated Urinary Tract Infection. Jpn J Infect Dis 2018;71:158-61.

13. Draft Guidance for Industry on Complicated Urinary Tract Infections: Developing Drugs for Treatment; Availability. U.S. Food \& Drug Administration Documents / FIND; Washington. Feb 24, 2012.

14. Blot S, Vandewoude K, De Bacquer D, et al. Nosocomial bacteremiacaused by antibiotic-resistant gram-negative bacteria in critically ill patients: clinical outcome and length of hospitalization. Clin Infect Dis 2002;34:1600-6.

15. Li L, Huang H. Risk factors of mortality in bloodstream infections caused by Klebsiella pneumonia: A singlecenter retrospective study in China. Medicine (Baltimore) 2017;96:e7924

16. Li L, Zhu C, Huang H. Clinical epidemiology and outcomes of biliary tract infections caused by Klebsiella pneumoniae. Ann Transl Med 2019;7:304.

17. Köves B, Magyar A, Tenke P. Spectrum and antibiotic resistance of catheter-associated urinary tract infections. GMS Infect Dis 2017;5:Doc06.

18. Gillen JR, Isbell JM, Michaels AD, et al. Risk factors for urinary tract infections in cardiac surgical patients. Surg Infect (Larchmt) 2015;16:504-8.

19. Hagerty T, Kertesz L, Schmidt JM, et al. Risk factors for catheter-associated urinary tract infections in critically ill patients with subarachnoid hemorrhage. J Neurosci Nurs 2015;47:51-4.

20. Saint S, Greene MT, Krein SL, et al. A program to prevent catheter-associated urinary tract infection in acute care. N Engl J Med 2016;374:2111-9.

21. Greene MT, Fakih MG, Fowler KE, et al. Regional variation in urinary catheter use and catheter-associated urinary tract infection: results from a national collaborative. Infect Control Hosp Epidemiol 2014;35:S99-106.

22. Goudie A, Dynan L, Brady PW, et al. Costs of Venous Thromboembolism, Catheter-Associated Urinary Tract Infection, and Pressure Ulcer. Pediatrics 2015;136:432-9.

23. Platt R, Polk BF, Murdock B, et al. Mortality associated with nosocomial urinary tract infection. $\mathrm{N}$ Engl J Med 1982;307:637-42.

24. Kuriyama A, Takada T, Irie H, et al. Prevalence and
Appropriateness of Urinary Catheters in Japanese Intensive Care Units: Results From a Multicenter Point Prevalence Study. Clin Infect Dis 2017;64:S127-30.

25. Li F, Song M, Xu L, et al. Risk factors for catheterassociated urinary tract infection among hospitalized patients: A systematic review and meta-analysis of observational studies. J Adv Nurs 2019;75:517-27.

26. Loveday HP, Wilson JA, Pratt RJ, et al. epic3: national evidence-based guidelines for preventing healthcareassociated infections in NHS hospitals in England. J Hosp Infect 2014;86 Suppl 1:S1-70.

27. Amelia A, Nugroho A, Harijanto PN. Diagnosis and Management of Infections Caused by Enterobacteriaceae Producing Extended-Spectrum b-Lactamase. Acta Med Indones 2016;48:156-66.

28. Castanheira M, Deshpande LM, Mendes RE, et al. Variations in the Occurrence of Resistance Phenotypes and Carbapenemase Genes Among Enterobacteriaceae Isolates in 20 Years of the SENTRY Antimicrobial Surveillance Program. Open Forum Infect Dis 2019;6:S23-33.

29. Ranjan Dash N, Albataineh MT, Alhourani N, et al. Community-acquired urinary tract infections due to extended-spectrum beta-lactamase-producing organisms in United Arab Emirates. Travel Med Infect Dis 2018;22:46-50.

30. Søraas A, Sundsfjord A, Sandven I, et al. Risk factors for community-acquired urinary tract infections caused by ESBL-producing enterobacteriaceae-a case-control study in a low prevalence country. PLoS One 2013;8:e69581.

31. US Food and Drug Administration, Center for Drug Evaluation and Research. Complicated urinary tract infections: developing drugs for treatment. Guidance for industry 2015.

32. Pallett A, Hand K. Complicated urinary tract infections: practicalsolutions for the treatment of multiresistant gramnegative bacteria. J Antimicrob Chemother 2010;65:iii25-33.

33. Sante L, Lecuona M, Aguirre Jaime A, et al. Risk factors to secondary nosocomial bacteremia to UTI in a tertiary hospital. Rev Esp Quimioter 2019;32:311-6.

34. Letica-Kriegel AS, Salmasian H, Vawdrey DK, et al. Identifying the risk factors for catheter-associated urinary tract infections: a large cross-sectional study of six hospitals. BMJ Open 2019;9:e022137.

Cite this article as: Liu X, Sai F, Li L, Zhu C, Huang H. Clinical characteristics and risk factors of catheter-associated urinary tract infections caused by Klebsiella Pneumoniae. Ann Palliat Med 2020;9(5):2668-2677. doi: 10.21037/apm-20-1052 